\title{
Development of a Home-based Wrist Rehabilitation System
}

\author{
Radzi Ambar ${ }^{1,}$, Muhammad Faiz Zakaria ${ }^{2}$, Muhammad Shukri Ahmad ${ }^{3}$, Siti Zarina Muji ${ }^{4}$, \\ Muhammad Mahadi Abd Jamil ${ }^{5}$ \\ ${ }^{1,2,4}$ Computational Signal, Imaging and Intelligence (CSII) Research Group, Department of Computer Engineering, \\ Universiti Tun Hussein Onn Malaysia, Malaysia \\ 1,2,3,5 Biomedical Modelling and Simulation (BIOMEMS) Research Group, Department of Electronic Engineering, \\ Universiti Tun Hussein Onn Malaysia, Malaysia \\ 1,2,3,4,5 Faculty of Electrical and Electronic Engineering, Universiti Tun Hussein Onn Malaysia, Malaysia
}

\begin{tabular}{l} 
Article Info \\
\hline Article history: \\
Received Sep 2, 2017 \\
Revised Nov 16, 2017 \\
Accepted Nov 30, 2017 \\
\hline
\end{tabular}

Keyword:

Accelerometer

Computer game

Home-based

Wrist rehabilitation system

\begin{abstract}
There are several factors that may result to wrist injuries such as athlete injuries and stroke. Most of the patients are unable to undergo rehabilitation at healthcare providers due to cost and logistic constraint. To solve this problem, this project proposes a home-based wrist rehabilitation system. The goal is to create a wrist rehabilitation device that incorporates an interactive computer game so that patients can use it at home without assistance. The main structure of the device is developed using $3 \mathrm{D}$ printer. The device is connected to a computer, where the device provides exercises for the wrist, as the user completes a computer game which requires moving a ball to four target positions. Data from an InvenSense MPU-6050 accelerometer is used to measure wrist movements. The accelerometer values are read and used to control a mouse cursor for the computer game. The pattern of wrist movements can be recorded periodically and displayed back as sample run for analysis purposes. In this paper, the usefulness of the proposed system is demonstrated through preliminary experiment of a subject using the device to complete a wrist exercise task based on the developed computer game. The result shows the usefulness of the proposed system.
\end{abstract}

Copyright $@ 2017$ Institute of Advanced Engineering and Science. All rights reserved.

\section{Corresponding Author:}

Radzi Ambar,

Departement of Computer Engineering,

Faculty of Electric and Electronic Engineering,

Universiti Tun Hussein Onn Malaysia, Malaysia.

Email: aradzi@uthm.edu.my

\section{INTRODUCTION}

A recent report by World Health Organization (WHO) has found that stroke and ischaemic heart disease are the world's top causes of death worldwide, makes up for a combined 15 million deaths in 2015 [1]. According to the National Stroke Association of Malaysia (NASAM), in Malaysia alone, over 50,000 new cases are reported each year [2]. The report also elaborated that stroke is the third main cause of mortality after heart disease and cancer, and the leading cause of adult disability. Majority of stroke survivors cannot have normal self-reliant lives because of the incapability to utilize their upper limbs for activities of daily living (ADL) such as grasping and feeding.

Various research articles stated that stroke patients are able to regain motor function with regular sessions of therapy and rehabilitation on the effected lower or/and upper extremities [3-5]. Studies have shown that active repetitive motions tasks can increase the strength of the affected limbs [6-8]. Rehabilitation procedures are based on clinical assessment tools which can be executed by self-report (home base) and observer-rated (done at rehabilitation centers) [9]. Observer-rated needs recurring visits to rehabilitation centers which is time consuming and costly due to the use of dedicated therapists. Furthermore, commonly 
used rehabilitation procedures involving conventional rehabilitation devices and one to one interaction with physiotherapist have been found to be ineffective, where 30 to $60 \%$ of patients unable to use their more affected arms functionally after discharged [10], [11].

Early discharge and home-based rehabilitation are the logical choice because patient integration with community can begin early. Moreover, home-based rehabilitation proved to stimulate good physical health due to its ability to permit motor and functional gains that occurred with natural recuperation and sense of happiness with community integration [12]. However, for caregivers related to home-based rehabilitation, they are exposed to the potential risk of poorer mental health condition [13]. On the other hand, on behalf of patients who prefer home-based rehabilitation, the costs for hiring caregivers at home are expensive. Therefore, how to successfully motivate patients to continuously perform physical exercises at home is a crucial research topic [14].

Previous studies on upper-limb rehabilitation devices have used pneumatic muscle driven devices [15], [16] and robotic exoskeletons [17-19]. Koeneman et al. developed a pneumatic muscle driven devices that are able to provide EMG biofeedback and force biofeedback to the user's wrist [15]. However, the device is limited to only flexion and extension motions. Most recently, Bartlett et al. introduced a pneumatically actuated orthosis in a form of soft wearable device that supports all the degree of freedom (DOF) of the wrist [16]. Although it is well received by a group of stroke patients due to the ability to assist in all wrist's DOF, the feedbacks on the convenient and comfort of wearing the device are unknown. On the other hand, robotic exoskeletons permits patients to receive varying repetitive rehabilitation treatment with the addition of various control strategies based on the patient's state [20]. But, exoskeletons usually consist of large and complex systems that are not suitable for home-based application.

Thus, this work describes the prototype design and preliminary experimental results of a low-cost and portable home-based wrist rehabilitation system that combines sensing technology with interactive computer game. The main aim of this work is to develop a user-friendly, safe, economical and more importantly fun rehabilitation system that can motivate patients to consistently undergo rehabilitation exercises at home or rehabilitation centers. The proposed system is consists of a 3D printed three mouse-like joystick that will be corresponded to flexion-extension (flex-ext), adduction-abduction (add-abd) and pronation-supination (pro-sup) motions once completed. However, as the device is still under development, add-abd motion is not available currently.

\section{DESCRIPTION OF THE WRIST REHABILITATION SYSTEM}

Figure 1 shows the overview of the proposed home-based wrist rehabilitation system that consists of a wrist rehabilitation device and interactive computer game. The developed wrist rehabilitation device contains an inertial measurement unit (IMU) MPU-6050 that consists of an accelerometer sensor that measures acceleration of wrist movements.

The acceleration raw data from MPU-6050 sensor are sent to an Arduino Leonardo microcontroller to be processed and mapped into angular data. The processed data will be transmitted to personal computer (PC), where the data will be used to play an interactive computer game developed using Unity game development software and Macro recorder software.

In the planned development of the computer game, the user will move the mouse cursor using the joystick, where Macro recorder software will save the exact movements of the cursor into text files. Using these text files, a dedicated Excel file will create run samples that can produce back the movements of the mouse cursor in $\mathrm{x}$ - and y-axes graphical images. Therefore, therapists can analyze the performance of rehabilitation based on these run samples that can be shown to the user. Therapists can efficiently alter exercise methods according to the performance and condition of the user.
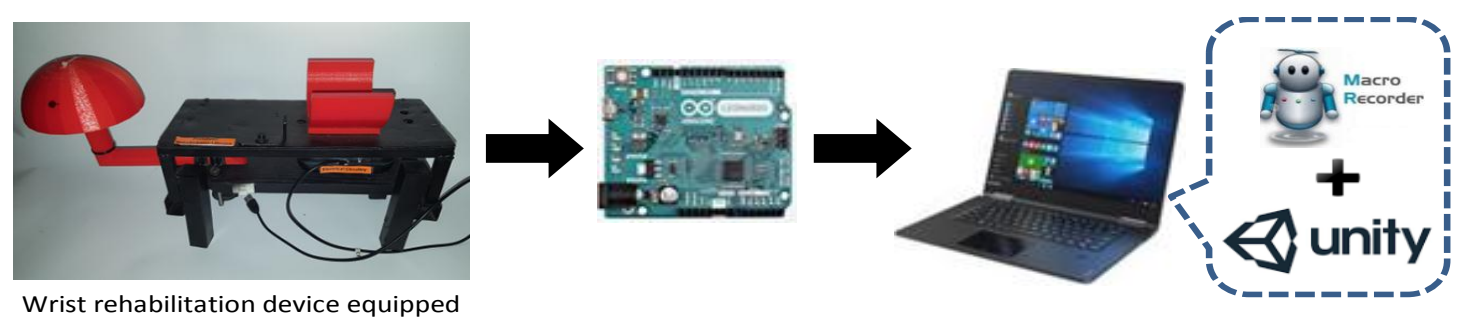
with MPU-6050 sensor

Figure 1. Overview of the proposed wrist rehabilitation system 


\subsection{Design Concept, Mechanical Parts, Materials and Manufacturing}

Taking into account the expensive cost to design robotic exoskeleton and also the convenient and comfort of wearing glove for rehabilitation, the design of the proposed device will be based on a single person mouse-like joystick. The reason to explore the applicability of mouse-like joystick is to take into consideration the inability of stroke patients to firmly grasp handle-like joystick. Mouse-like joystick concept will enable users to just lightly grasp the joystick surface to move it.

Figure 2 shows various actual images for the prototype of the developed wrist rehabilitation device. The prototype shown in the figure uses two types of wrist motions, flex-ext and abd-add. As shown in the figure, the base of the prototype is made of steel shafts and wooden pallet from recycled parts which cost nothing. The base has two rows, the lower row is for storing the electrical circuit, and the top row is for installing an LCD and a handle that connected to the mouse-like joystick.

The red coloured parts shown in Figure 2 are made of Acrylonitrille-Butadiene Styrene (ABS) material using a single-extruder MakerBot's Replicator 2X 3D printer. The parts include the mouse-like joystick which is the dome-like part, and a U-shaped part that is for supporting the forearm while using the joystick. The scale of the design is suitable for all ages. The idea of the joystick design is to enable free wrist motion and prevent any restriction on patient's wrist movement during rehabilitation. The run samples of the motions of the hand and wrist will be used to show the performance of the rehabilitation in the final analysis. Figure 3(a) shows the 3D printed parts designed using Sketchup software during the initial stage of development. Figure 3(b) shows the completed 3D printed parts. The dome-shaped joystick and handle parts are made into two halves. The parts are then assembled into a single part using screws and nuts as shown in Figure 2(a).

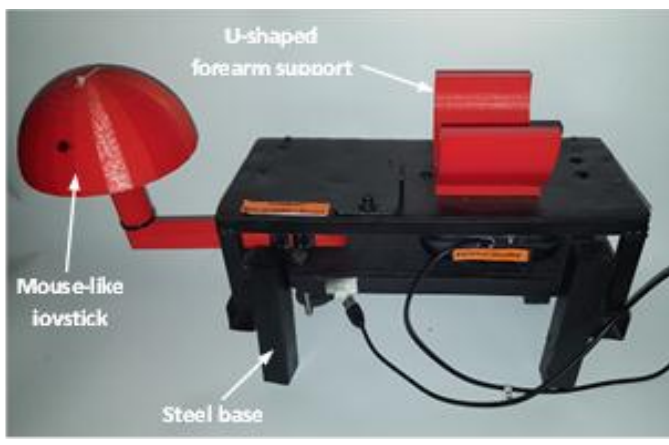

(a)

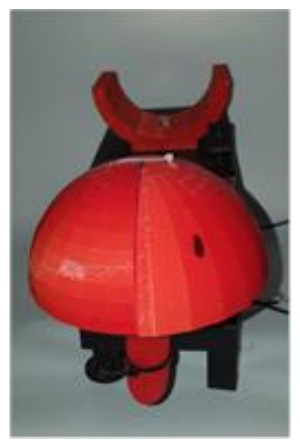

(b)

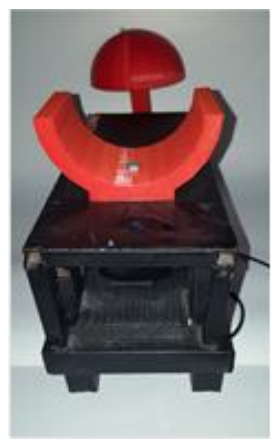

(c)

Figure 2. Overview of the proposed wrist rehabilitation system

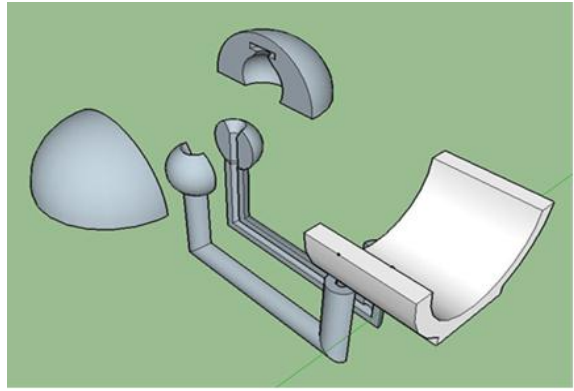

(a)

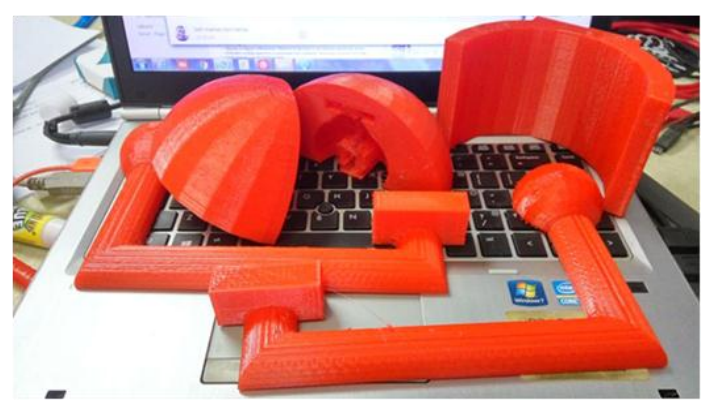

(b)

Figure 3. (a) 3D design of the mouse-like joystick using Sketchup software, (b) the completed 3D printed parts

Table 1 shows the type of motions that can be done using the developed wrist rehabilitation device. The table also shows the muscles that correspond to the type of wrist movement. Figure 4(a) and (b) shows the location of muscles and ligaments related to ext-flex and pro-sup motions, respectively. 
Table 1. Muscles that correspond to the type of wrist movement

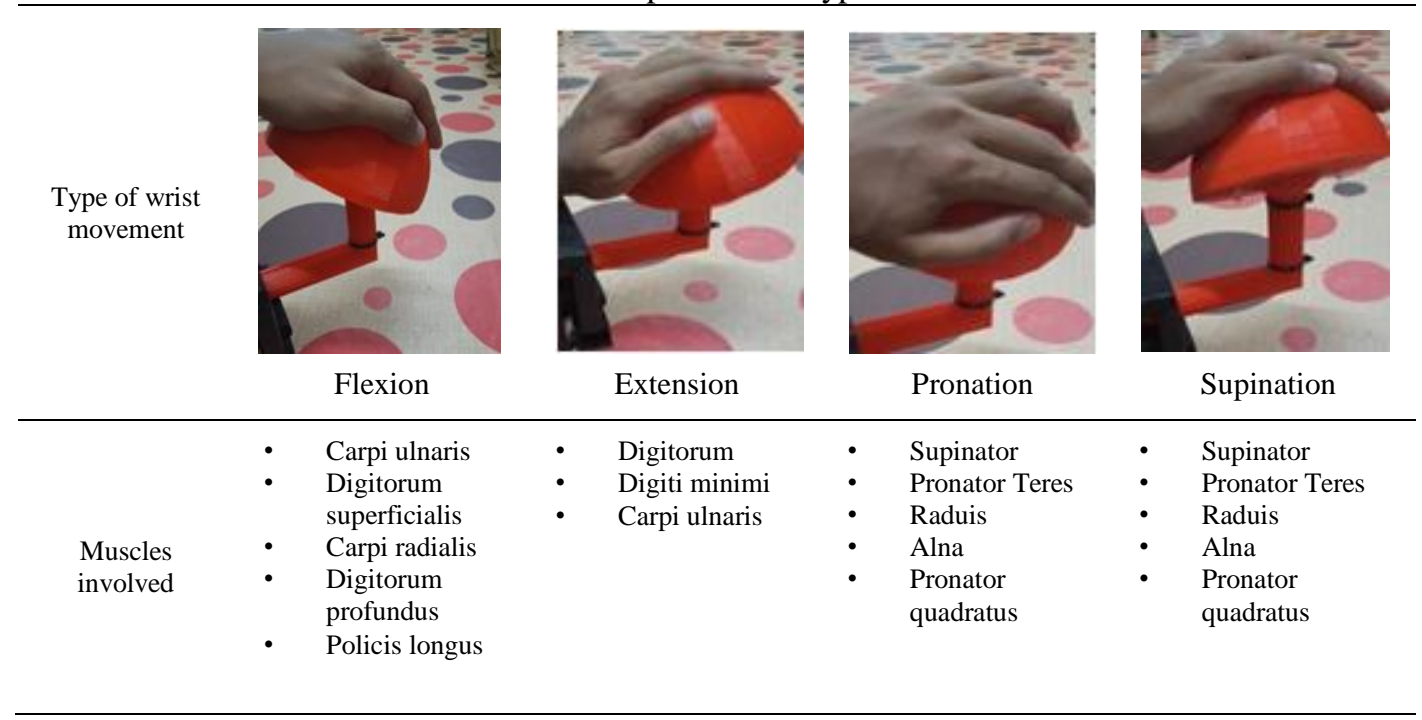

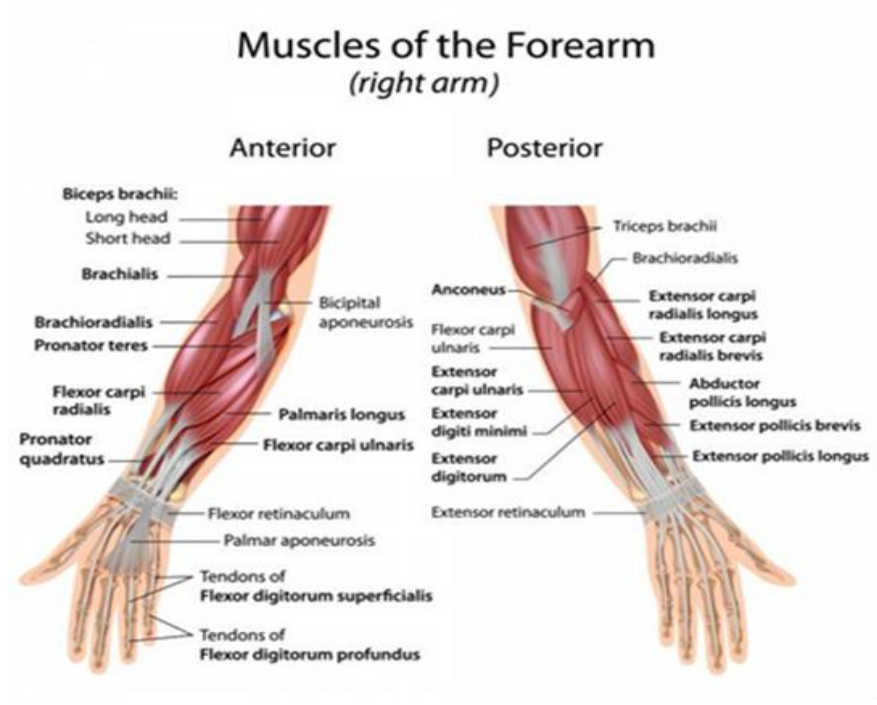

(a)

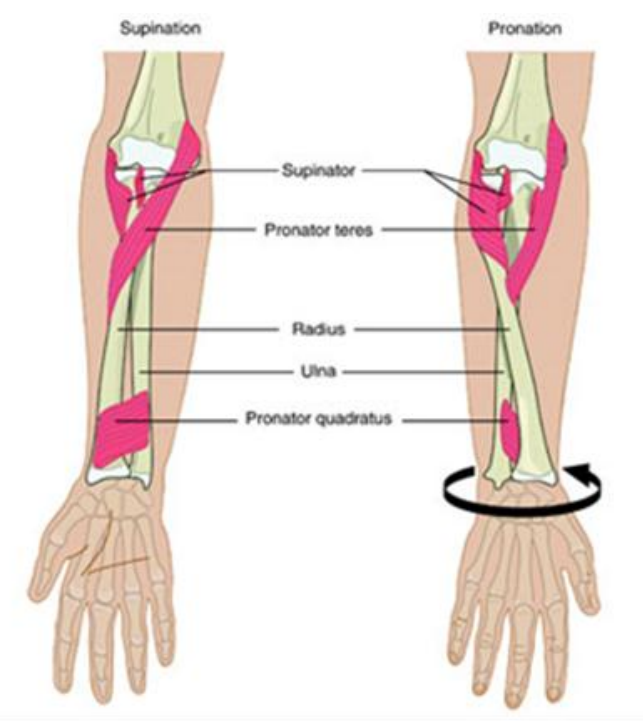

(b)

Figure 4. Muscles and ligaments related to (a) flex-ext motions [22], (b) sup-pro motions [23]

\subsection{Electrical Circuitry and Sensory Parts}

An Arduino Leonardo microcontroller is used in this project. Prior to selecting Arduino Leonardo, other types of microcontroller were initially considered including Arduino UNO and Mega. However, with the same clock speed $(16 \mathrm{MHz})$, Leonardo costs the less (costs USD22.10) compared to UNO (costs USD22.80) and Mega (costs USD43.00). Furthermore, an advantage of Leonardo is, it uses an ATmega32U4 as its microcontroller, where the board can emulate a computer mouse using USB protocol which is a requirement for this project.

A unit of IMU MPU-6050 from InvenSense is used in this project. The MPU-6050 sensor contains a Micro-Electro-Mechanical Systems (MEMS) accelerometer and a MEMS gyro in a single chip. It is known to be very accurate, as it contains 16-bits analog to digital conversion hardware for each channel/axis [21]. Therefore it can measure the $\mathrm{x}-, \mathrm{y}-$, and $\mathrm{z}$-axes simultaneously. The sensor uses the I2C-bus to interface with the Arduino. However, we only use the accelerometer data in the MPU-6050 to measure the acceleration generated from wrist's movements. 
Table 2 shows the pin connection between the MPU-6050 sensor (accelerometer only) and the Leonardo board. Figure 5(a) upper shows the CAD model of MPU-6050 connected to an Arduino Leonardo designed using Fritzing software. Figure 5(a) lower shows the actual Leonardo board that is connected to the MPU-6050 as shown in Figure 5(b). Figure 5(b) shows half of the 3D printed part for the mouse-like joystick has a space to attach the MPU-6050 sensor. Furthermore, the 3D printed handle part also has a narrow long space enough to hide sensor's cables. Actually, both halves of the 3D printed joystick and handle have spaces to hide the cables and sensors that will be hidden once both halves are assembled together and tightened using screws and nuts.

Table 2. Pin connection between MPU-6050 and Arduino Leonardo

\begin{tabular}{ccc}
\hline No & Pins on MPU-6050 sensor & Pins on Arduino Leonardo \\
\hline 1 & VCC & GV \\
2 & GND & GND \\
3 & SDA & Digital 2 \\
4 & SCL & Digital 3 \\
\hline
\end{tabular}

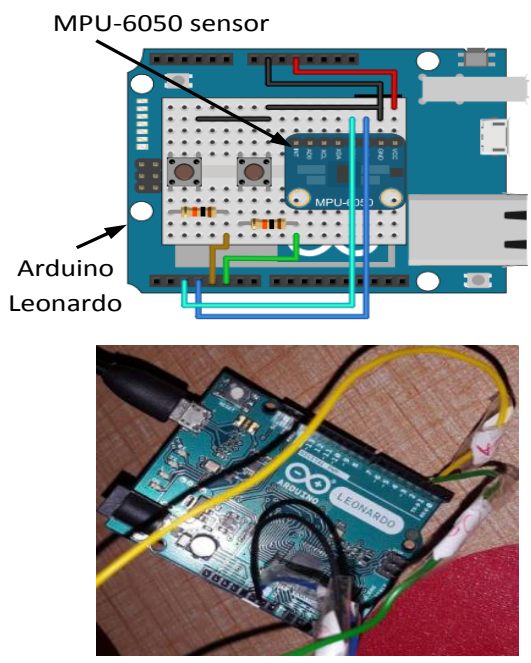

(a)

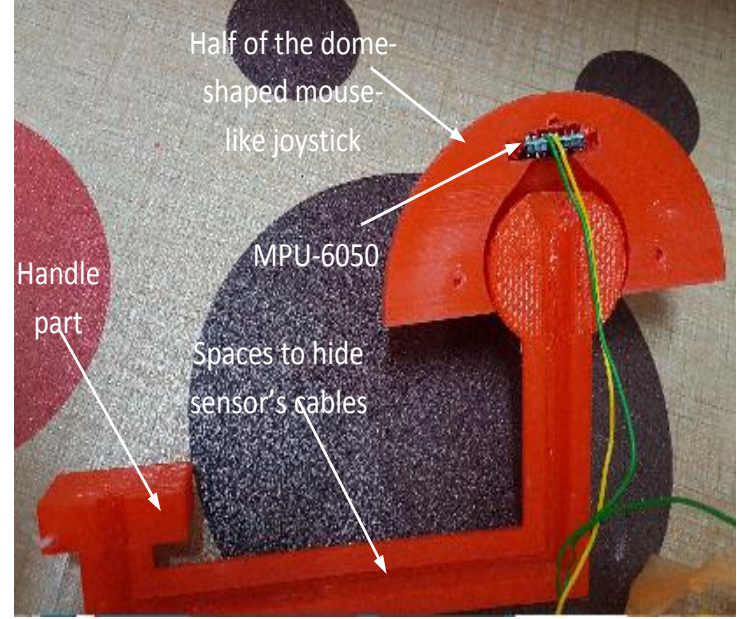

(b)

Figure 5. (a) Upper: CAD model of MPU-6050 connected to Arduino Leonardo. Lower: actual image of Arduino Leonardo, (b) MPU-6050 location inside the joystick. Spaces for hiding cables can be seen.

\section{INTERACTIVE COMPUTER GAME DEVELOPMENT}

\subsection{Connecting MPU-6050 and Arduino Leonardo to Unity}

The reason why Arduino Leonardo board is chosen in this work is due to the board can emulate a computer mouse using USB protocol. An IMU MPU-6050 sensor is utilized in this work that functions as a computer mouse to play an interactive computer game. As Arduino Leonardo uses an ATmega23U4 chip, it contains mouse and keyboard libraries that permit the board to function as a mouse or keyboard to a connected computer. Therefore, instead of using an actual mouse, the mouse cursor on the computer screen can be controlled using the MPU-6050 instead. In order to develop a computer game using Unity software to interact with any Arduino board, a user will need to purchase a Unity asset (ARDUnity) which costs about USD50 [24]. Thus, buying an Arduino Leonardo is a good option that can reduce the cost of developing the prototype.

Figure 6 shows a screenshot of the source code for Arduino Leonardo to control a mouse cursor using MPU-6050 sensor. In the source code, mpu.getMotion6(\&ax, \&ay, \&az, \&gx, \&gy, \&gz) means read six (6) values from MPU-6050 sensor into six (6) variables, namely ax, ay, az, gx, gy and gz. The first six (6) variables are for accelerations on $\mathrm{x}-, \mathrm{y}-$ and $\mathrm{z}$-axes that are used in this project. The rests are values from gyrosensor. Then, the acceleration raw values on $\mathrm{x}$ - and $\mathrm{y}$-axes which ranges between -16000 to 16000 are simply mapped between 90deg to-90deg. Finally, these values are then modified to distance values for moving the mouse cursor using the syntax Mouse.move(). 


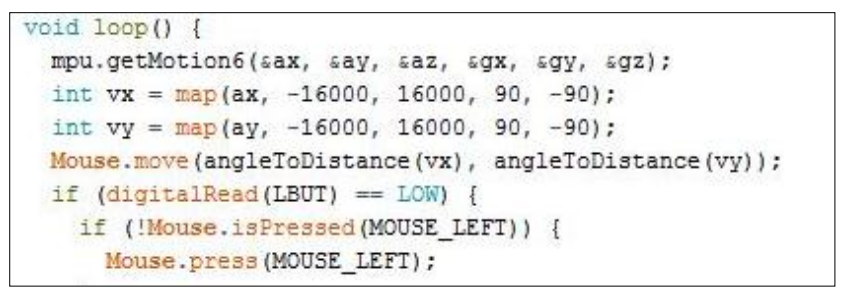

Figure 6. Screenshot of the source code for Arduino Leonardo controlling a mouse cursor

\subsection{Computer Game Development using Unity Software}

Unity is a C++-based game engine and the source codes can be written in C\# and JavaScript (UnityScript). Figure 7 shows a screenshot of the developed interactive computer game. In this game, the ball at the center is moved using the distance value from MPU-6050 sensor which is installed in the developed wrist rehabilitation device. The methods to derive the sensor values have been explained in the previous section. The user will be tasked to move the ball to four targets (goal post). Additionally, the user must move the ball on a straight line (blue lines as guides) to each of the targets. This subsection will explain the development of the game.

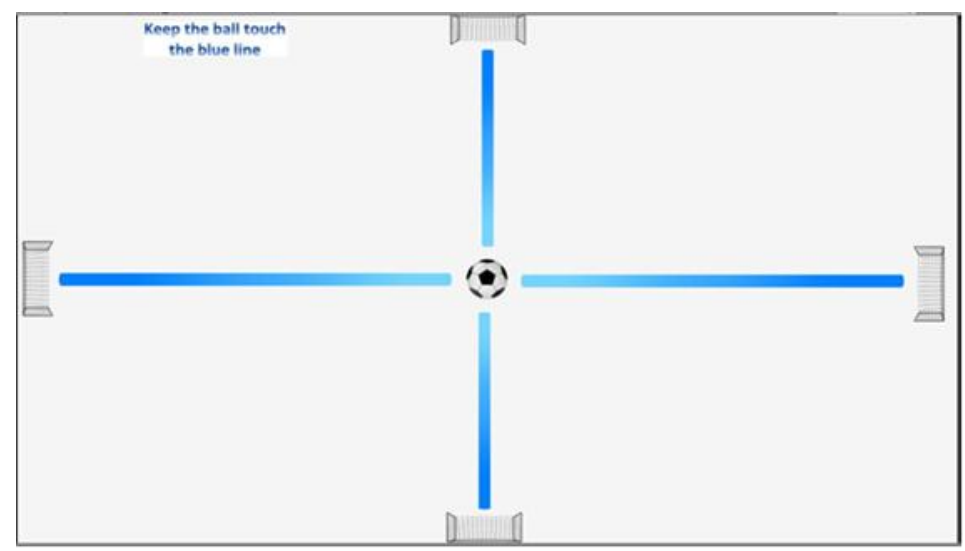

Figure 7. Screenshot of the developed interactive computer game

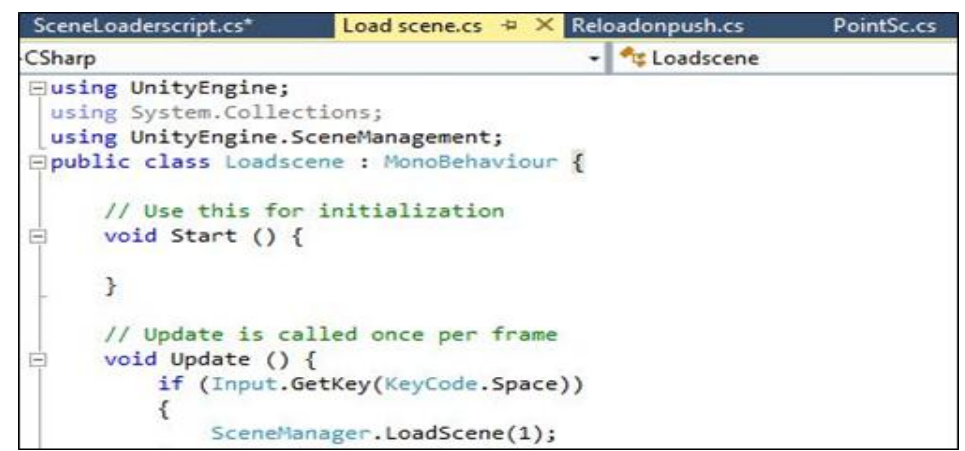

Figure 8. Screenshot of the Load scene.cs source code for Arduino Leonardo controlling a mouse cursor

To develop the interactive computer game, four (4) source codes using C++ and one (1) java script have been developed to make this game simple and easy to use. The first code for Unity is Load scene.cs, which is used to load the game from the menu. Figure 8 shows a screenshot of Load scene.cs. From the figure, the syntax Input.GetKey(KeyCode.Space) means the game will be loaded after user press the space key. 
The next source code is BallSc.cs as shown in Figure 9. This code is used to move the ball based on the movement of the mouse cursor. Here, ball is a GameObject in Unity. GameObjects are the basic components for Unity games. In Unity, every GameObjects need a source code attach to it to provide instructions. The source code BallSc.cs is attached to the ball in order to make this game work. From Figure 9, the Input.mousePosition is the important line in BallSc.cs which means the ball is moved based on mouse position. Thus, we can control the ball in the game by using MPU-6050 sensor installed in the wrist rehabilitation device.

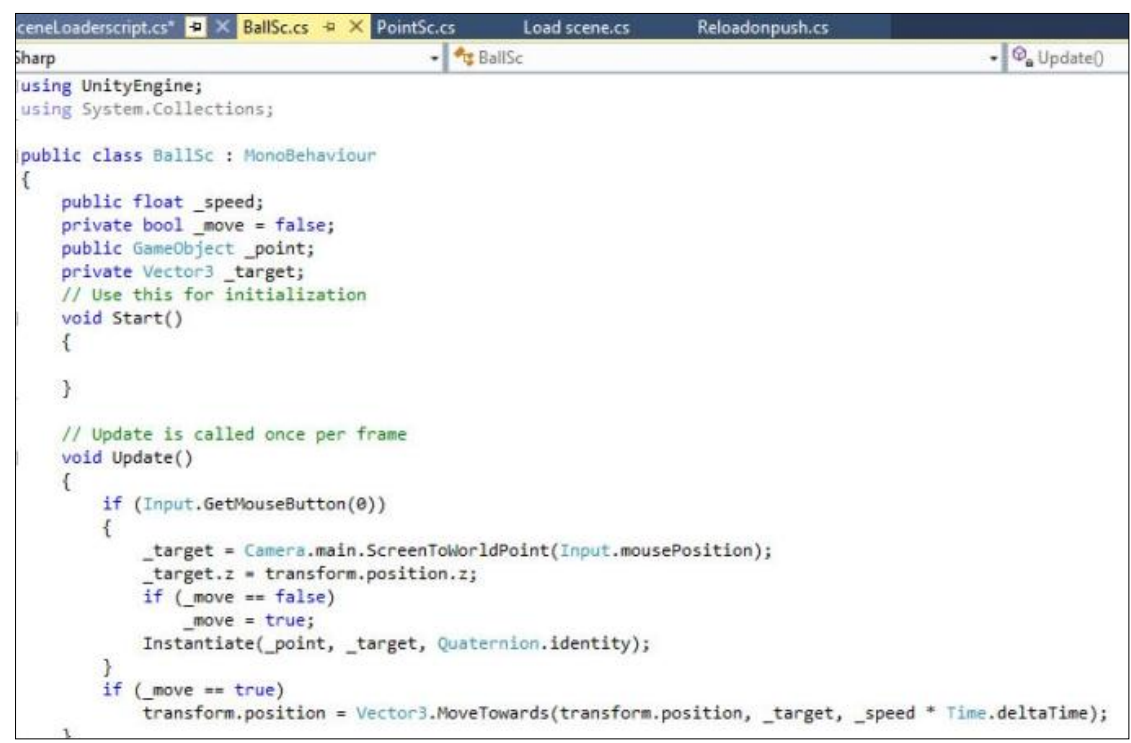

Figure 9. Screenshot of the BallSc.cs source code for controlling ball movement using mouse cursor

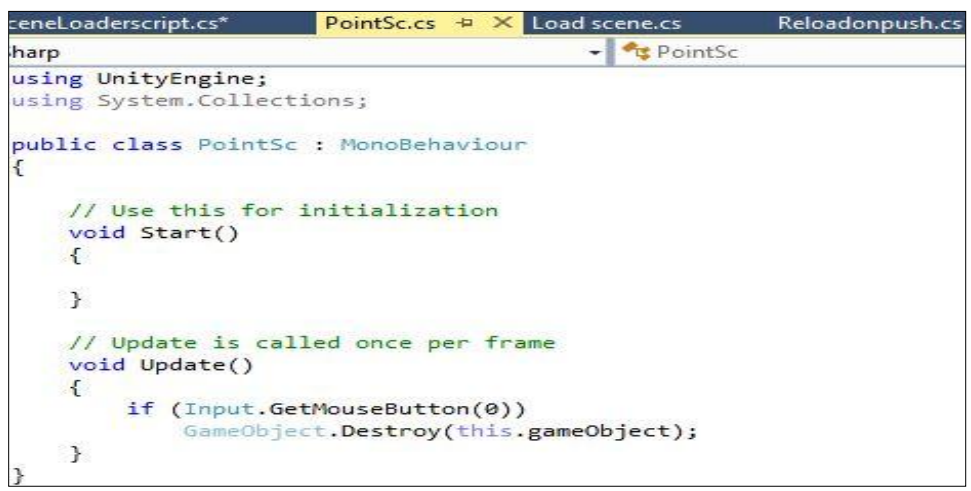

Figure 10. Screenshot of the PointSc.cs source code for moving the ball based on mouse click

Figure 10 is PointSc.cs source code which is just a simple code to show that the user can move the ball based on mouse click by using red dot game object. In this code, Input.GetMouseButton( 0$)$ means that if the left click is pressed, the red dot will appear which means this game is ready to play.

MPU-6050 sensor is a very sensitive sensor. Therefore, we need to center the mouse cursor before playing the game. So, Reloadonpush.cs is a source code that reset the mouse position by centering ball position with the mouse cursor as shown in Figure 11. In the code, Input.GetKeyDown(KeyCode.LeftShift) means it will reset after press left shift key.

Figure 12 shows the final code developed using Java script code for quitting the game. As shown in Figure 12, the code is showing quit to home screen when the users press the right click. After compiling all the source code with the game object, the rehabilitation computer will be displayed as in Figure 7. 


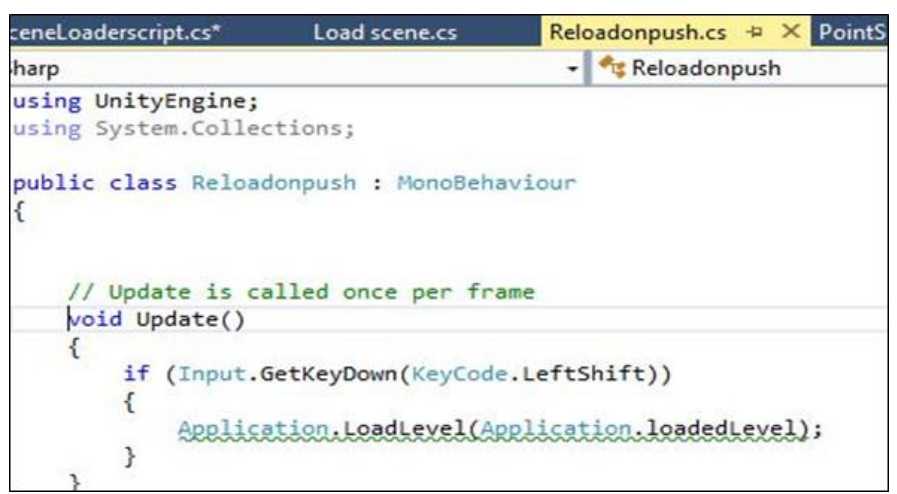

Figure 11. Screenshot of the Reloadonpush.cs source code for reset the mouse position by centering ball position with the mouse cursor

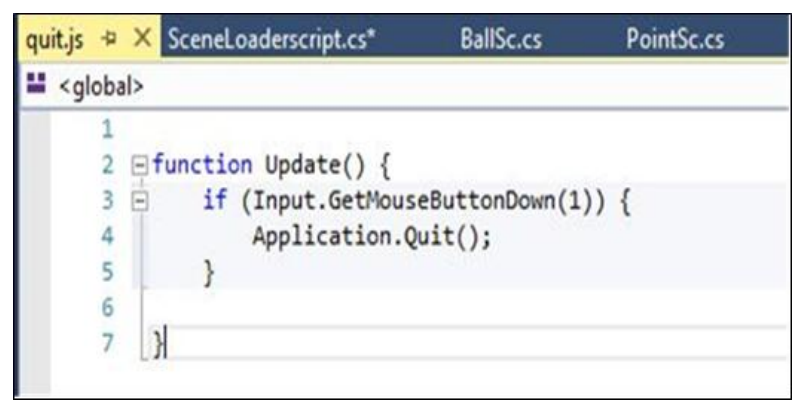

Figure 12. Java code for quitting the game

\section{EXPERIMENTAL RESULTS AND DISCUSSIONS}

In this section, we will describe the preliminary experimental results of this study. A right handed healthy subject was asked to complete a task using the developed computer game (Figure 7) which require moving a ball to four target positions using the developed wrist rehabilitation device shown in Figure 2 . The data of wrist movements were recorded for analysis purposes. Figure 13 shows the movements of the ball to be completed by the subject. The subject was required to move the ball at the center according to the numbered arrows starting from arrow (1) until arrow (8).

Figure 14 shows a sample run of a video game done by the subject based on Figure 13 requirement. As shown in Figure 14, the subject started the movement of the ball from the center to the right end of the goal post which is the first target position. Then, the subject returned back the ball to the center position referring to arrow number (2) in Figure 13. Next, the subject moved the ball from the center to the goal post on the downward position referring to arrow number (3). Then, returned back to the center position referring to arrow number (4). The subject continued to move the ball until the last arrow number (8), where the end position is labelled as in Figure 14.

Based on the sample run in Figure 14, the healthy subject had successfully moved the ball according to the required trajectory shown in Figure 13 which demonstrates encouraging results of the proposed wrist rehabilitation device. The sample runresult shows that the trajectories of the ball movement done by the healthy subject are smooth, close to the blue line and nearly in a straight line on each target position. This result is expected from a healthy subject that has no wrist disability that can significantly disturb or influence hand movements. However, as the dome-shaped controller is made from 3D printed material, the internal overall surface including internal part is rough. Therefore, the trajectory is somehow affected by the roughness of the surface which was confirmed by the user. For future work, the rough surface will be made smoother by sanding using sand paper.

Nevertheless, the authors assumed that the trajectories perform by patients with wrist and hand disability will be significantly different where unsmooth trajectories will be produced. Furthermore, further tests will be carried out by voluntary patients to show the effectiveness of the proposed device. Comparison of the results obtained from healthy subjects with the voluntary patients will be made to demonstrate the usefulness of the system. 


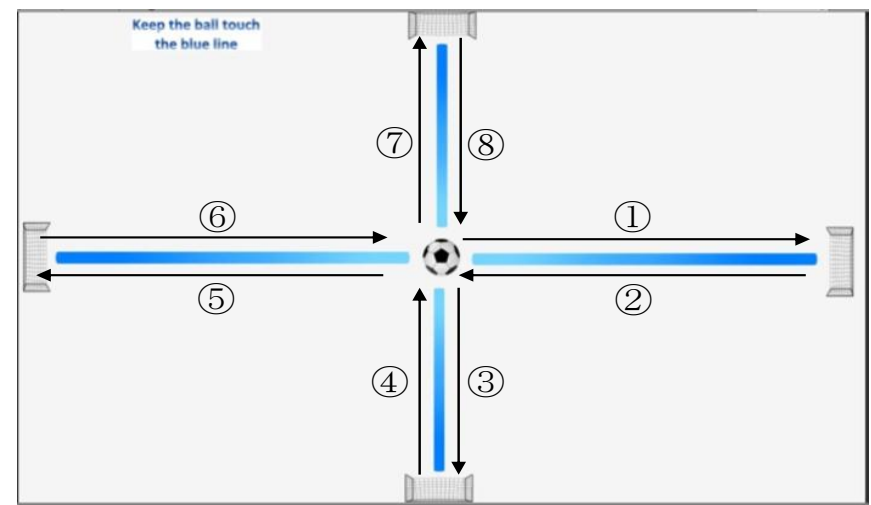

Figure 13. Subject is required to move the ball to four target positions according to the arrows

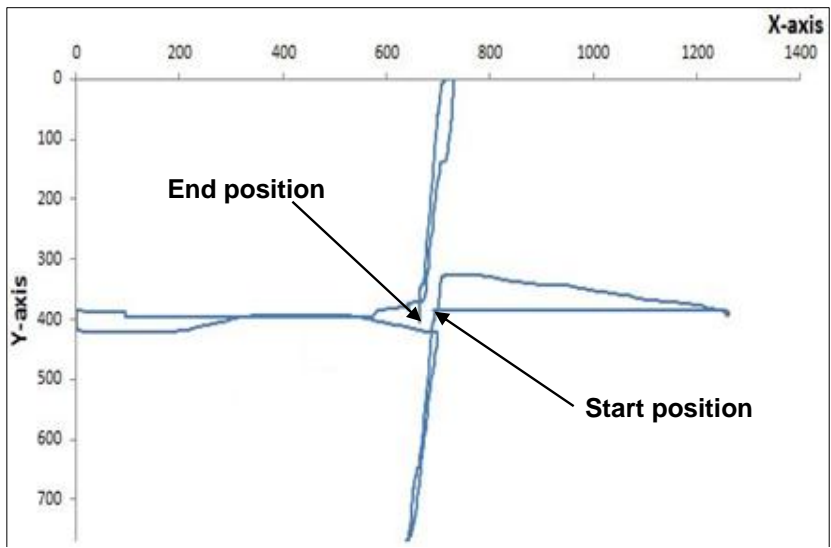

Figure 14. Run sample result of the motions done by a subject

\section{CONCLUSION}

In conclusion, a home-based wrist rehabilitation system by incorporating interactive computer game has been proposed to help the user or patient that have wrist problem to do rehabilitation at home without therapist assistance. In this work, detail explanation of the developed wrist rehabilitation device and interactive computer game have been presented. Preliminary experimental results show the usefulness of the proposed device where the movement of the rehabilitation device installed with the IMU sensor enables a subject to control an object to move to every target positions. Currently, the developed device can only measure wrist motions on extension-flexion and pronation-supination only. Therefore, in the future, the device will be upgraded to support measurement for adduction-abduction motions. Furthermore, the design of the device will be improved by making it smaller using 3D printer utilizing ABS material. The possibility of using the device with games application on smartphone will be explored.

\section{REFERENCES}

[1] "The top 10 causes of death" [Online]. Available:http://www.who.int/mediacentre/factsheets/fs310/en/. [Accessed: 25-May-2017].

[2] "National Stroke Association of Malaysia" [Online]. Available: http://www.peoplegiving.org/nasam. [Accessed: 25-May-2017].

[3] S. L. Wolf, C. J. Winstein, J. P. Miller, E. Taub, G. Uswatte, D. Morris, C. Giuliani, K. E. Light, D. Nichols-Larsen et al., "Effect of constraintinduced movement therapy on upper extremity function 3 to 9 months after stroke," JAMA: the Journal of the American Medical Association, vol. 296, no. 17, pp. 2095-2104, 2006.

[4] B. Kibler, "Shoulder rehabilitation: principles and practice," Clinical Supplement: The Shoulder, vol 30, 1998, pp. 40-50.

[5] R. Donatelli, "Physical Therapy of the Shoulder," Clinics in Physical Therapy, 2003.

[6] C. E. Levy, D. S. Nichols, P. M. Schmalbrock, P. Keller, and D. W. Chakeres, "Functional MRI evidence of cortical reorganization in upper-limb stroke hemiplegia treated with constraint-induced movement therapy," Am J Phys Med Rehabil, vol. 80, pp. 4-12, 2001. 
[7] J. Liepert, I. Uhde, S. Graf, O. Leidner, and C. Weiller, "Motor cortex plasticity during forced-use therapy in stroke patients: a preliminary study," J Neurol, vol. 248, pp. 315-21., 2001.

[8] H. Johansen-Berg, H. Dawes, C. Guy, S. M. Smith, D. T. Wade, and P. M. Matthews, "Correlation between motor improvements and altered fMRI activity after rehabilitative therapy," Brain, vol. 125, pp. 2731-42., 2002

[9] R. Ambar, M. S. Ahmad and M. M. Abdul Jamil, "Design and Development of a Multi-sensor Monitoring Device for Arm Rehabilitation," Int. J. of Integrated Engineering, vol. 3, no. 2, pp. 55-62, 2011.

[10] B. Brewer, S. McDowell, and L. Worthen-Chaudhari, "Poststroke upper extremity rehabilitation: a review of robotic systems and clinical results," Topics in stroke rehabilitation, vol. 14, no. 6, pp. 22-44, 2007.

[11] P. W. Duncan, "Synthesis of intervention trails to improve motor recovery following stroke," Top Stroke Rehabil 3: 1-20, 1997.

[12] N. E. Mayo et al., "There's No Place Like Home : An Evaluation of Early Supported Discharge for Stroke," Stroke, 31(5):1016-1013, 2000.

[13] C. Anderson et al., "Home or hospital for stroke rehabilitation? results of a randomized controlled trial : I: health outcomes at 6 months," Stroke, 31(5):1024-1031, 2000.

[14] J. Eriksson, M. J. Mataric and C. J. Weinstein, "Hands-off Assistive Robotics for Post-Stroke Arm Rehabilitation," In Proc. IEEE 9th International Conference on Rehabilitation Robotics, pp. 22-24, 2005.

[15] E. J. Koeneman, R. S. Schultz, S. L. Wolf, D. E. Herring and J. B. Koeneman, “A pneumatic muscle hand therapy device," Proc. of IEEE Eng. in Medicine and Biology Society, vol. 4, pp. 2711-2713, 2004.

[16] N. W. Bartlett et al., "A Soft Robotic Orthosis for Wrist Rehabilitation,” J. Med. Devices, 9(3), 030918, 2015.

[17] H. I. Krebs, N. Hogan, M. L. Aisen, and B. T. Volpe, "Robot-aided neurorehabilitation," IEEE Trans. Rehab. Eng., vol. 6, no. 1, pp. 75-87, 1998.

[18] C. G. Burgar, P. S. Lum, P. C. Shor, and H. F. Machiel Van der Loos, "Development of robots for rehabilitation therapy: the Palo Alto VA/Stanford experience," J Rehabil Res Dev, vol. 37, no. 6, pp. 663-73, 2000.

[19] J. Oblak, I. Cikajlo, and Z. Matjacic, "Universal haptic drive: A robot for arm and wrist rehabilitation," IEEE Transactions on Neural Systems and Rehabilitation Engineering, vol. 18, no. 3, pp. 293-302, 2010.

[20] L. Marchal-Crespo and D. J. Reinkensmeyer, "Review of control strategies for robotic movement training after neurologic injury," Journal of Neuroengineering and Rehabilitation, vol. 6, no. 1, p. 20, 2009.

[21] "MPU-6050Accelerometer+Gyro"[Online].Available:http://playground.arduino.cc/Main/MPU-6050. [Accessed: 25-May-2017].

[22] "More Insight Into Developing Grip Strength: Your Hand Digits" [Online]. Available:https://breakingmuscle.com/learn/more-insight-into-developing-grip-strength-your-hand-digits. [Accessed: 25-May-2017].

[23] "Explore Points Therapy, Ocupational Therapy, and more"[Online]. Available: https://www.pinterest.com/pin/391179917613620666 [Accessed: 25-May-2017]

[24] "What is ARDUnity Asset?" [Online]. Available: https://sites.google.com/site/ardunitydoc/ [Accessed:25-May2017]

\section{BIOGRAPHIES OF AUTHORS}
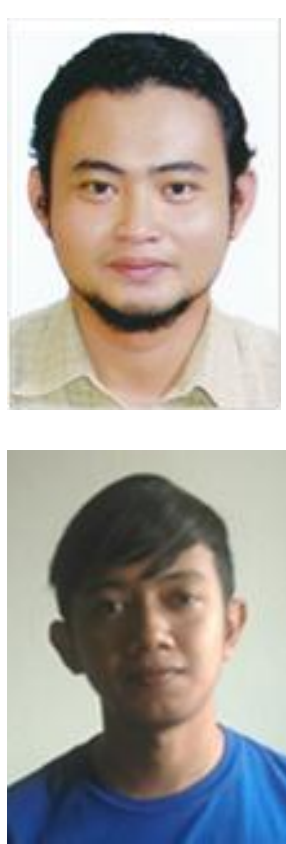

Radzi Ambar received his Diploma in Control and Information Systems Engineering from Tsuruoka National College of Technology, Japan in 2001, and Bachelor of Engineering in Information and Computer Science Engineering, from Toyohashi University of Technology, Japan in 2003. Shortly afterwards he worked as a Software Engineer for 4 years at Panasonic Communication Malaysia. Then, he joined Sharp Manufacturing Malaysia as a Quality Assurance Engineer from 2008 to 2009. He acquired his MSc. degree in Electrical Engineering in 2011 from Universiti Tun Hussein Onn Malaysia. He received his Ph.D in Mechanical and Control Engineering (Robotics) from Kyushu Institute of Technology, Japan in 2015. At present he is a senior lecturer at the Faculty of Electrical and Electronic Engineering at Universiti Tun Hussein Onn Malaysia. His research interests include robotics engineering and assistive devices for rehabilitation.

Muhammad Faiz Zakaria received his Bachelor of Electronics and Electrical Engineering (Computer Engineering) with Honours in 2017. He is currently an intern in Ericool Sdn. Bhd., Sungai Petani. His research interests include assistive devices for rehabilitation and embedded systems. 


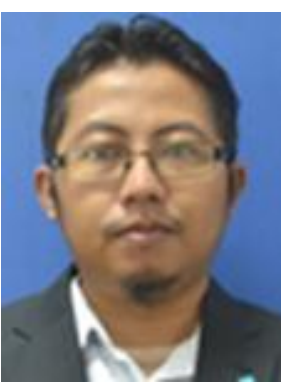

Muhammad Shukri Ahmad completed his Bachelor of Electrical Engineering (Computer Engineering) and Master of Electrical Engineering (Microelectronics) from Kolej Universiti Tun Hussein Onn (now Universiti Tun Hussein Onn Malaysia) in 2002 and 2004 respeectively. He is currently working as a Lecturer in Electronic Engineering Department, Faculty of Electrical and Electronic Engineering, Universiti Tun Hussein Onn Malaysia. His research interest is on Internet of Things and Medical Rehabilitation Apparatus Design.

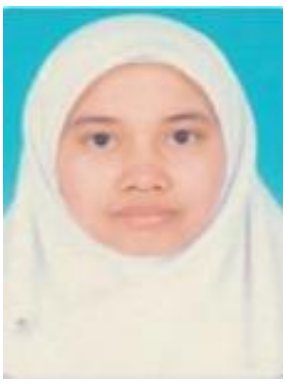

Siti Zarina Mohd. Muji received her M.Sc. degree in Electric and Electronic Engineering from Universiti Sains Malaysia in 2004 and his Ph.D degree in Electronic Instrumentation at Universiti Teknologi Malaysia in 2012. She is currently an Associate Professor at the Faculty of Electric and Electronic Engineering, Universiti Tun Hussein Onn Malaysia, focusing on Optical Tomography and application.

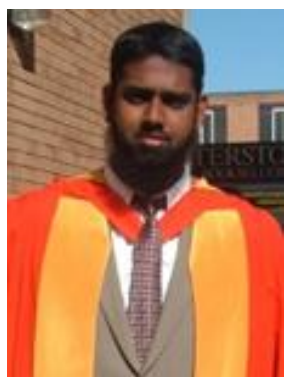

Muhammad Mahadi Abdul Jamil completed his Diploma in Medical Electronics Engineering from British Malaysian Institute, Kuala Lumpur, Malaysia and then acquired his first degree in Medical Engineering at University of Bradford, United Kingdom. He completed his Ph.D degree from Bradford University working on the biomedical/ bioassay applications of high resolution widefield surface plasmon microscopy. Currently, he is working as an Associate Professor at Universiti Tun Hussein Onn Malaysia. For the past 8 years he has been actively conducting research on the medical electronic, biomedical and bioelectronics engineering. He also has special interest in research areas such as rehabilitation monitoring devices, sports performance technology, microfabrication and cell engineering. To date he has attained several national research grants from the Ministry of Higher Education Malaysia 